\title{
Choroidal Detachment after Uncomplicated Small Incision Cataract Surgery
}

\author{
Chikako Suto $^{a, b}$ Satoru Mita ${ }^{a, b}$ Sadao Hori ${ }^{a}$ \\ a Department of Ophthalmology, Tokyo Women's Medical University School of \\ Medicine, Tokyo, and ${ }^{b}$ Department of Ophthalmology, Saiseikai Kurihashi \\ Hospital, Saitama, Japan
}

\section{Key Words}

Choroidal detachment - Cataract surgery - Angiotensin II receptor blocker . Telmisartan $\cdot$ Sub-Tenon anesthesia

\begin{abstract}
Background and Purpose: Angioedema is a well-recognized side effect of angiotensinconverting enzyme inhibitors, but is rarely associated with angiotensin II receptor blockers (ARB). Here, we report the first case of a patient on ARB therapy (telmisartan) for hypertension who developed serous choroidal detachment localized to the posterior pole after sub-Tenon anesthesia for small incision cataract surgery.

Methods and Results: An 82-year-old Japanese woman who received oral medications for hypertension underwent cataract surgery with sub-Tenon anesthesia using $2 \%$ Xylocaine $^{\circledR}$ on her left eye. Her corrected distance visual acuity improved to 20/25 on the first day after the surgery. On the fifth day, however, it decreased to 20/40 and choroidal detachment was detected at the posterior pole. We suspected an increase of choroidal vascular permeability and started oral steroid therapy. After 1 week, the area of detachment was smaller and her acuity improved to $20 / 20$. Subsequently, she underwent cataract surgery without sub-Tenon anesthesia on her right eye, and no choroidal detachment occurred.

Conclusion: This is the first published case of ARB-induced choroidal detachment after uncomplicated small incision cataract surgery. Sub-Tenon anesthesia may aggravate angioedema associated with ARB therapy, so ophthalmologists should be aware of this rare complication.
\end{abstract}

\section{Introduction}

Telmisartan (Micardis ${ }^{\circledR}$; Boehringer Ingelheim, Ridgefield, Conn., USA; Astellas, Tokyo, Japan) is an orally active angiotensin II receptor blocker (ARB) used for the 
treatment of hypertension. ARBs are generally a safe alternative for patients who cannot tolerate angiotensin-converting enzyme (ACE) inhibitors. The incidence of angioedema associated with ACE inhibitor therapy ranges from 0.1 to $1 \%$, whereas the reported incidence of ARB-induced angioedema ranges from 0.1 to $0.4 \%$ [1]. Fromberg et al. [2] reported that a patient on ACE inhibitor therapy for hypertension and glomerulonephritis developed circumferential choroidal detachment (CD) after uncomplicated cataract surgery. Serous CD generally runs continuously from the pars plana or the ora serrata around the eyeball to the equator, and it is rarely confined to the posterior pole [3]. Here, we report on a hypertensive patient on ARB therapy who developed CD localized to the posterior pole after uncomplicated small incision cataract surgery with sub-Tenon anesthesia.

\section{Case Report}

An 82-year-old Japanese woman had been diagnosed with hypertension 3 years earlier. She was initially treated with the calcium channel blocker amlodipine besylate (Norvasc ${ }^{\circledR}$; Pfizer Inc., New York, N.Y., USA) at a dose of $5 \mathrm{mg}$ /day. Telmisartan $40 \mathrm{mg} /$ day was added by her local physician to achieve better blood pressure control 1 year after amlodipine treatment had been started. She had no known allergies such as to penicillin, aspirin, or nonsteroidal anti-inflammatory drugs (NSAIDs). She had not received ACE inhibitors before and there was no history of angioedema in herself or her family members.

In April 2009, bilateral age-related cataracts were diagnosed and cataract surgery was planned for her left eye with implantation of a one-piece acrylic intraocular lens (SN60WS; Alcon Inc., Fort Worth, Tex., USA). The axial length of her right eye was $25.12 \mathrm{~mm}$ and that of her left eye was $24.73 \mathrm{~mm}$, as measured with an IOLMaster ${ }^{\circledR}$ (Carl Zeiss Meditec, Jena, Germany). A 2.75-mm superior corneoscleral incision was made after $1.0 \mathrm{ml}$ of $2 \%$ Xylocaine ${ }^{\circledR}$ had been injected with a blunt $27-\mathrm{G}$ needle from 1 o'clock for sub-Tenon anesthesia. Then, uncomplicated small incision cataract surgery was performed. Acetazolamide was not administered.

On the first day after the surgery, there was a deep anterior chamber with mild inflammation and good self-sealing wound closure, and the intraocular pressure was $14 \mathrm{~mm} \mathrm{Hg}$. Her corrected distance visual acuity (CDVA) had improved to 20/25. An antibiotic (moxifloxacin; Vegamox ${ }^{\circledR}$ ) and betamethasone (Rinderon ${ }^{\circledR}$ ) was started topically 4 times/day. On the fifth day after the surgery, her CDVA was decreased to 20/40 and the intraocular pressure was $15 \mathrm{~mm} \mathrm{Hg}$. There was optic capture of the intraocular lens and CD was seen at the posterior pole on mydriatic examination (fig. 1a), even though there had been no postoperative trauma. Computed tomography showed serous CD but no nanophthalmos, scleral hypertrophy, or a tumor (fig. 2). Fluorescein angiography confirmed choroidal folds at the site of $\mathrm{CD}$, but there were no significant findings. Indocyanine green angiography revealed a slight increase in the permeability of choroidal vessels at the site of CD (fig. $1 \mathrm{~b}, \mathrm{c})$. Therefore, we diagnosed inflammatory CD and started oral steroid treatment. After 1 week, her CD showed a decrease in both area and thickness, while her CDVA improved to 20/20. Subsequently, cataract surgery was performed on her right eye under topical anesthesia while steroid therapy was continued, and CD did not occur. Her steroid therapy was tapered within a 2-week period. After 2 months, choroidal folds were still detected, but the CD had resolved. Three months after the surgery, the choroidal folds also disappeared and her CDVA improved to 20/16. Indocyanine green angiography demonstrated normal permeability of choroidal vessels at the previous site of CD.

\section{Discussion}

To our knowledge, this is the first case of CD localized to the posterior pole caused by sub-Tenon anesthesia in a hypertensive patient on ARB therapy undergoing uncomplicated small incision cataract surgery. In fact, the cause of angioedema 
associated with ARB therapy is not completely understood. ARBs are usually considered to be a safe alternative to ACE inhibitors since the serum level of bradykinin is unlikely to be affected. However, angioedema has also been reported in patients taking ARBs, suggesting that alternative pathways or mechanisms may be involved in this adverse reaction. Although not proven in humans, one possible explanation is that secondary stimulation of angiotensin II receptors causes an increase in tissue bradykinin which then results in angioedema [4].

Known causes of $\mathrm{CD}$ include a low intraocular pressure associated with eye surgery (e.g. after trabeculetomy and intra- or extracapsular cataract extraction [5]), blunt trauma, inflammatory diseases (e.g. scleritis or Harada disease), intraocular tumors, uveal effusion syndrome, and treatment with acetazolamide [6]. Angioedema is caused by various drugs, including penicillin, aspirin, NSAIDs [7], ACE inhibitors [1, 2, 8], and ARBs [1]. Except for ARB therapy, the patient had none of these predisposing factors.

Occurrence of $\mathrm{CD}$ at the posterior pole may have been associated with increased permeability of the choroidal vessels caused by localized inflammation. Transient elevation of intraorbital pressure induced by sub-Tenon anesthesia may stimulate the sclera around the injection site [9] and could cause local inflammation that reaches the choroidal layer. In addition, injection of Xylocaine near the vorticose veins may disturb the choroidal circulation. These factors could facilitate an increase of vascular permeability in the choroid associated with ACE inhibitor or ARB therapy. After vascular permeability increases, proteins will fail to pass through the filters of Bruch's membrane and the retinal pigment epithelium, while plasma will enter the suprachoroidal space and causes CD [10]. This mechanism could account for the occurrence of CD localized to the injection site after sub-Tenon anesthesia.

Hypersensitivity is unlikely since the patient showed no allergic reaction to Xylocaine before the surgery.

In summary, sub-Tenon anesthesia is an easy and safe method with good efficacy that is widely used for ophthalmologic surgery, but we should remember that it can sometimes cause choroidal folds or CD as in the present case. In particular, sub-Tenon anesthesia can aggravate angioedema associated with ACE inhibitor or ARB therapy, so ophthalmologists should be aware of this rare complication.

\section{Disclosure Statement}

None of the authors have a conflict of interest to disclose. 


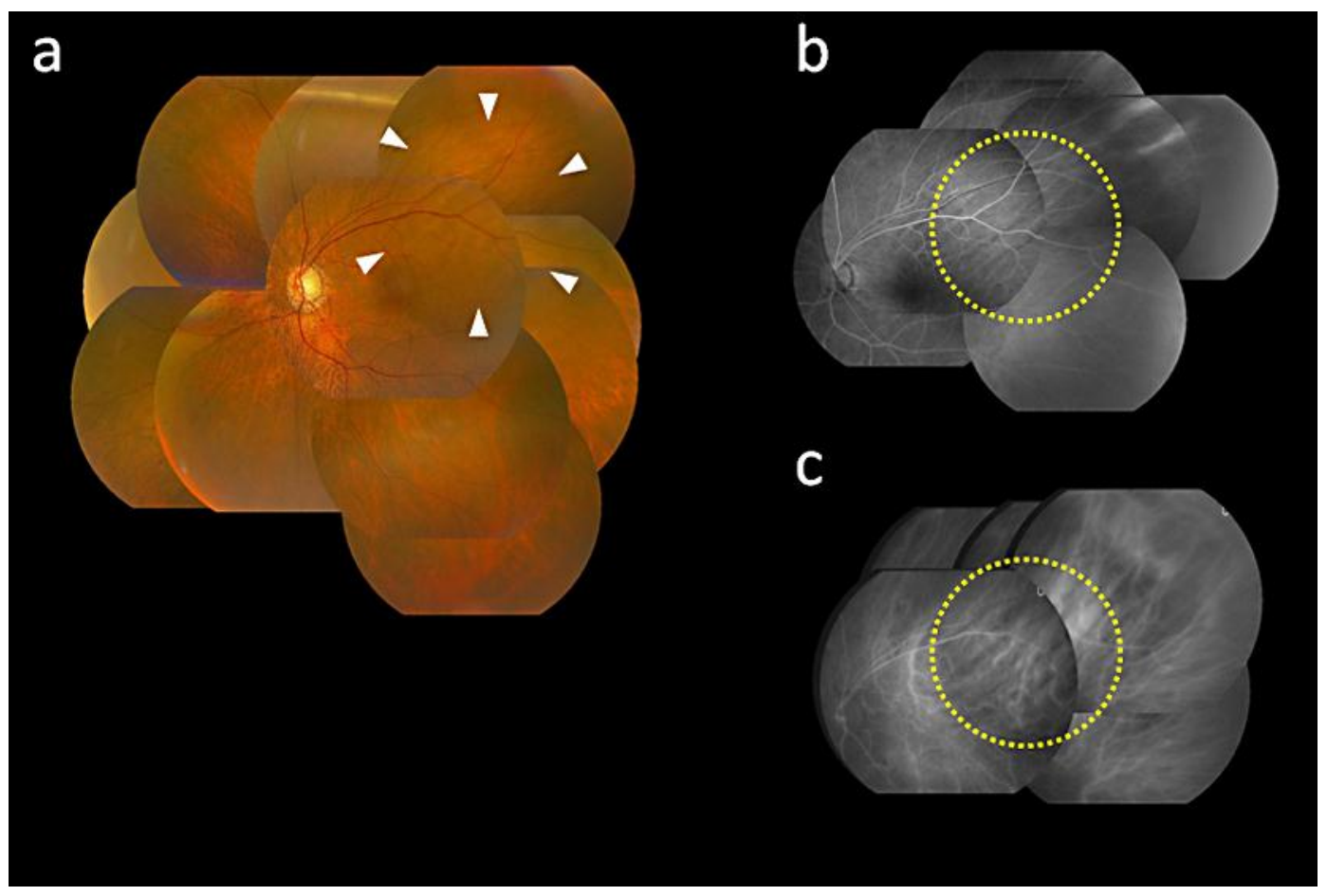

Fig. 1. a Photograph of the left eye showing CD at the posterior pole (arrowheads). b Fluorescein angiography of the left eye showing choroidal folds (yellow circle). c Indocyanine green angiography showing slightly increased permeability of choroidal vessels in the lesion (yellow circle).
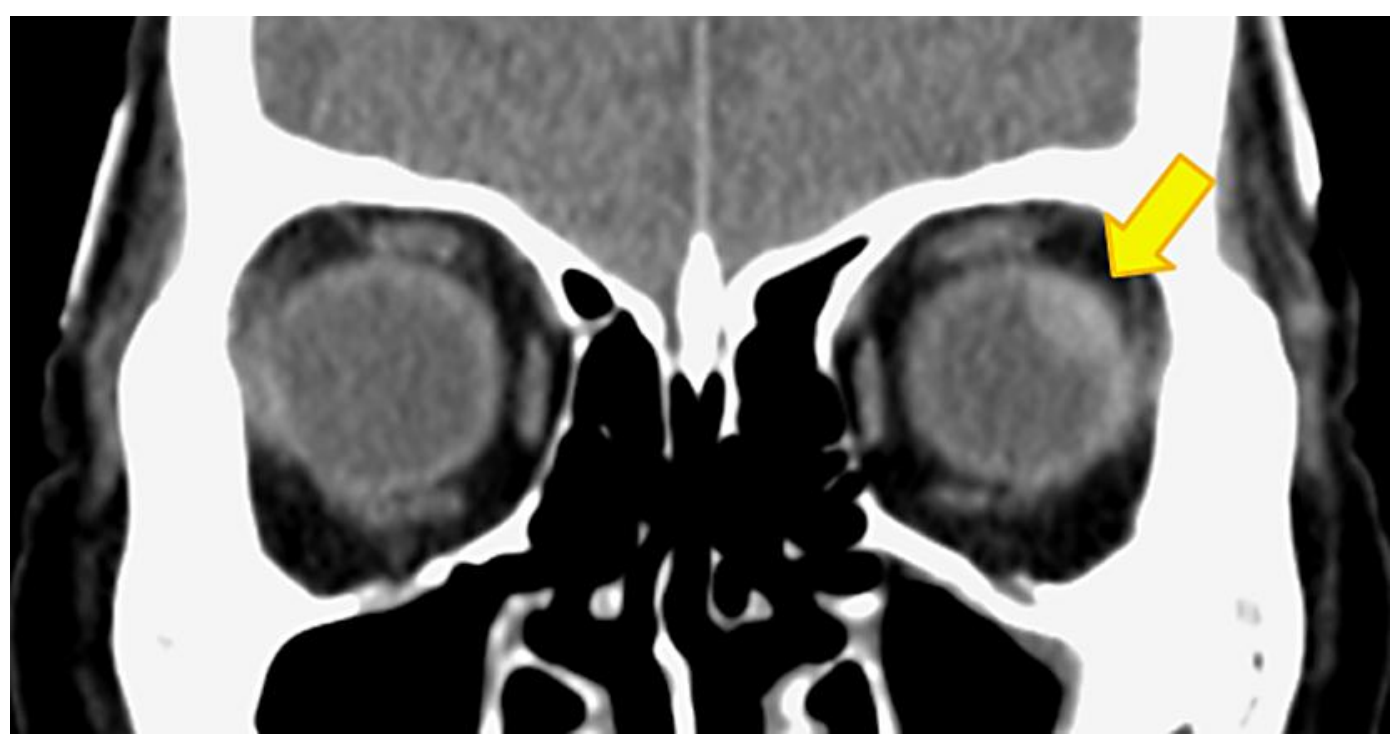

Fig. 2. Computed tomography of the left eye showing serous CD (arrow). 


\section{References}

1 Dickstein K, Kjekshus J: Effects of losartan and captopril on mortality and morbidity in high-risk patients after acute myocardial infarction: the OPTIMAAL randomised trial. Optimal Trial in Myocardial Infarction with Angiotensin II Antagonist Losartan. Lancet 2002;360:752-760.

-2 Fromberg I, Krause M, Brückner K, Lang M, Seitz B: Choroidal effusion after uncomplicated cataract surgery [in German]. Klin Monbl Augenheilkd 2008;225:591-593.

-3 Brubaker RF, Pederson JE: Ciliochoroidal detachment. Surv Ophthalmol 1983;27:281-289.

4 Irons BK, Kumar A: Valsartan-induced angioedema. Ann Phamacother 2003;37:1024-1027.

-5 Swyers EM: Choroidal detachment immediately following cataract extraction. Arch Ophthalmol 1972;88:632-634.

6 Parthasarathi S, Myint K, Singh G, Mon S, Sadasivam P, Dhillon B: Bilateral acetazolamide-induced choroidal effusion following cataract surgery. Eye 2007;21:870-872.

$>7$ Knowles SR, Drucker AM, Weber EA, Shear NH: Management options for patients with aspirin and nonsteroidal anti-inflammatory drug sensitivity. Ann Pharmacother 2007;41:1191-1200.

8 Reed LK, Meng J, Joshi GP: Tongue swelling in the recovery room: a case report and discussion of postoperative angioedema. J Clin Anesth 2006;18:226-229.

9 Nakaizumi A, Minami M, Sato T, Ishizaki E, Maeno T, Ikeda T: Four cases of choroidal fold induced by Tenon's capsule anesthesia during vitreous surgery [in Japanese]. Japanese Journal of Ophthalmic Surgery 2007;20:541-544.

10 Bellows AR, Chylack LT Jr, Hutchinson BT: Choroidal detachment. Clinical manifestation, therapy and mechanism of formation. Ophthalmology 1981;88:1107-1115.

Presented in part as a poster at the Japanese Society of Ophthalmic Surgeons, Tokyo, Japan, 2010. 